\title{
COMPETENCIAS INTERCULTURALES EN LA SOCIEDAD DIGITAL DEL CONOCIMIENTO
}

\author{
Olga Camila Hernández* Franklin Arciniegas**
}

\section{RESUMEN}

El artículo recopila los resultados parciales de una investigación con relación al desarrollo de habilidades lingüísticas y competencias interculturales. Proporciona en tres etapas una mirada hacia el desarrollo y utilización de las competencias digitales por parte de los estudiantes del programa de Lenguas Modernas de la ECCI. Mediante la indagación de antecedentes y estudios realizados en otros contextos, se vinculan las dos temáticas centrales de la investigación: las competencias digitales y las competencias interculturales. Se recurrió a la implementación de instrumentos de recolección de datos que permitieran acercarse a la población seleccionada para identificar las competencias claves en cuanto a la gestión compleja del conocimiento, en relación con las tecnologías y el desarrollo intercultural de los estudiantes de lenguas modernas. Finalmente, a través de una herramienta digital (video-juego), se auscultó la integración de competencias en TIC en los los planes de estudios, de tal manera que se pudiera potenciar el desarrollo del lenguaje a través de una vivencia intercultural.

\section{PALABRAS CLAVE}

Competencias digitales, conocimiento, educación, Interculturalidad, lenguaje, video-juego. 


\begin{abstract}
This article collects the partial results of a research that provides a look into three steps toward the development and use of digital skills by students in the program of Modern Languages of the $\mathrm{ECCl}$ in relation to the development of linguistic skills and intercultural competences. Through background checks and studies in other contexts that link the two central themes of this research which are: digital and intercultural competences; implementing data collection instruments that allow to approach the target population, identifying the key competencies for complex knowledge management in relation to technologies and intercultural development of students of modern languages, and finally the integration of ICT skills in the curriculum through a digital tool (video game), which may enhance their language development through an intercultural experience.
\end{abstract}

\title{
KEYWORDS
}

Digital competences, education, interculturality, knowledge, language, videogame.

\section{INTRODUCCIÓN}

La sociedad del siglo XXI se conoce como la sociedad del conocimiento. La transformación que se ha experimentado en los últimos dos siglos se ha producido por múltiples factores, entre los cuales se pueden destacar el proceso de globalización, el impacto de las tecnologías de la información y la comunicación, y la administración del conocimiento.

Un nuevo escenario se perfila con nuevos entornos de trabajo y de aprendizaje. La concepción de Internet se ha revolucionado durante los últimos años, gracias a lo que se conoce como la Web 2.o. Quienes la utilizan, ya no son meros espectadores o consumidores de la información que provee la red, pues, de manera sencilla, se pueden convertir en protagonistas de la misma, avanzando un paso más para ser sujetos activos que pueden crear conocimiento, publicarlo y compartirlo con otros.

En ese contexto emerge la necesidad de una alfabetización que posibilite alcanzar las habilidades y competencias digitales, de manera que se pueda considerar a las personas como sujetos alfabetizados digitalmente. Se requiere, además, que dicho proceso sirva para utilizar esos conocimientos y manejarse airosamente en distintas situaciones sociales, por lo cual ha de ser una alfabetización activa.

El ambiente educativo actual está estrechamente relacionado con los procesos de apertura económica, globalización del conocimiento y flexibilización de las fronteras culturales. Bajo esta visión, el aprendizaje de lenguas extranjeras, por ejemplo el inglés, se presenta como una herramienta de acercamiento a elementos socio culturales y disciplinares que enriquecen el saber y el ser y que posibilitan la diversificación de la práctica educativa.

En concordancia con lo descrito, uno de los retos de la educación superior en este siglo es generar sistemas educativos más pertinentes a las realidades y necesidades culturales de la población destinataria, razón por la cual, uno de los principales objetivos de la presente investigación es ahondar en la comprensión de las competencias del siglo XXI para los estu- 
diantes universtarios. Con ello se pretende mejorar la calidad de los procesos educativos y fortalecer el perfil de los profesionales egresados.

La competencia digital debe ser una prioridad en lo que a las estrategias de aprendizaje permanente se refiere, ya que las TIC se están convirtiendo en un elemento cada vez más importante para el ocio, el aprendizaje y el trabajo en todos los ámbitos. En la actualidad, el concepto de competencia digital se ha reformado con la aparición y el uso de nuevas herramientas de informática social, que dan lugar a nacientes habilidades relacionadas con la colaboración, intercambio, apertura, reflexión y formación de la identidad. También con los desafíos, tales como la calidad de la información, la confianza, responsabilidad, privacidad y seguridad.

El presente proyecto se realiza con el objetivo principal de identificar competencias claves para la gestión compleja del conocimiento en relación con las tecnologías y el proceso intercultural de los estudiantes de lenguas modernas, mediante una cartografía de dichas competencias. El ejercicio permitirá, de manera dinámica, la inclusión de las TIC en el currículo. De este modo, se evita que el uso de la tecnología sea producto de una facultad de algunos estudiantes para pasar a convertirse en instrumento al alcance de todos, incrementando la comprensión tecnológica de los alumnos mediante la integración activa de competencias en TIC en los planes de estudios.

En aras de llevar a cabo el proyecto, se diseñó una investigación planeada en tres fases. Una primera de conceptualización, para crear los elementos necesarios que ayuden a la construcción teórica del documento. La segunda está ligada a la recopilación de la información y el comienzo de un proceso de análisis de las necesidades que evidencian los estudiantes, tanto en el manejo de herramientas digitales como en la gestión de conocimiento que realizan a través de esos medios. Durante la fase tres se pretende la creación de un elemento de aprendizaje, un serious game, que ayude no solo a adquirir las habilidades en el manejo de las herramientas tecnológicas sino que contribuya al desarrollo de competencias interculturales y comunicativas, con la pretención de una formación integral de los estudiantes. Para la tercera fase del proyecto se ha utilizado una investigación descriptiva, ya que se busca identificar las competencias pertinentes a las necesidades de los estudiantes de Lenguas modernas de la Escuela Colombiana de Carreras Industriales - ECCI.

\section{OBJETIVOS}

- Identificar competencias claves para la gestión compleja del conocimiento, en relación con las tecnologías y el desarrollo intercultural de los estudiantes de lenguas modernas, para el logro de un perfil del educando, acorde con los requerimientos de la sociedad del conocimiento.

- Construir una cartografía de las competencias digitales y de las interrelaciones entre los distintos procesos implicados en el desarrollo de las mismas.

- Diseñar instrumentos que posibiliten medir las competencias de manera no lineal sino integrada y que puedan dar cuenta de procesos cognitivos creativos, colaborativos, de resolución de problemas, entre otros.

- Incrementar la comprensión tecnológica de los estudiantes mediante la integración en los planes de estudios - los currículos- de competencias en TIC, con un enfoque de nociones básicas de tecnología, a través de un video-juego pedagógico.

\section{Elementos conceptuales}

La educación, como componente fundamental de la sociedad, no ha sido ajena a los efectos del avance de las nuevas tecnologías. Aunque han sido muchos los esfuerzos por seguir paralelamente su desarrollo, en algunos casos lo que se ha propiciado es la repetición del modelo tradicional; es decir, las múltiples 
herramientas de las cuales pueden disponer los jóvenes se ven limitadas a su manipulación, lo que que no garantiza el aprendizaje significativo.

En efecto, el proyecto propone la creación de un modelo de competencias interculturales que puedan potenciar el desarrollo de competencias comunicativas en una segunda lengua; asimismo, el uso de las TIC como instrumento cognitivo y para la interacción y colaboración grupal.

Se basa en que esta herramienta tecnológica brinda unas funciones que se hacen vitales en la educación y garantizan el libre acceso a la información, la que luego se verá transformada en conocimientos si la universidad y la escuela crean espacios propicios para el proceso de investigación.

Por lo tanto, es plausible que la participación en redes digitales favorezca la aceptación y la valoración positiva de la pluralidad, referida no solo a las diferencias que puedan existir por razones culturales o de género sino a la diversidad intrínseca que es propia como seres particulares.

\section{Elementos metodológicos}

Desde el punto de vista metodológico, se pueden recorrer diferentes caminos para indagar la realidad social a partir de paradigmas como el cuantitativo y el cualitativo. Cada enfoque tiene su propia fundamentación epistemológica, diseños metodológicos, técnicas e instrumentos acordes con la naturaleza de los objetos de estudio, las situaciones sociales y las preguntas que se plantean los investigadores, ya sea con el propósito de explicar, comprender o transformar la realidad social. En ese marco, el proyecto que se expone se desarrolla con un enfoque cualitativo, debido a las características del análisis, pues se busca explorar un contexto específico, identificar y describir las competencias digitales y su relación con las competencias interculturales.

El enfoque cualitativo por lo común, se utiliza primero para descubrir y refinar preguntas de investigación. A veces, pero no necesariamente, se prueban hipótesis.
Con frecuencia se basa en métodos de recolección de datos sin medición numérica, como las descripciones y las observaciones. (Hernández, 2006, p. 5).

En concordancia con lo anterior, el tipo de investigación propuesta es descriptiva, pues esta investigación consiste en conocer las situaciones, costumbres y actitudes predominantes a través de la descripción exacta de las actividades, objetos, procesos y personas. Su meta no se limita a la recolección de datos sino a la predicción e identificación de las relaciones que existen entre dos o más variables.

Los investigadores no son meros tabuladores; ellos recogen los datos sobre la base de una hipótesis o teoría, exponen y resumen la información de manera cuidadosa y luego analizan minuciosamente los resultados, a fin de extraer generalizaciones significativas que contribuyan al conocimiento. La gráfica 1. muestra la ruta metodológica de la segunda fase de la investigación.

\section{Investigación cualitativa de tipo descriptivo}

POBLACIÓN: Estudiantes lenguas
modernas de la Ecci Muestra: 6o
Estudiantes (1- -8 - 9 ) semestre
VARIALES: Competencias digitales,
competencias interculturales
\[ \begin{array}{l}\text { Técnicas de recolección de datos: Observación, } \\ \text { análizis documental, entrevista. } \\ \text { Instrumentos: Formatos de cuestionario, guías } \\ \text { de entrevista, grabadora } \\ \text { Técnicas de anàlisis de datos: } \\ \text { lógicas (deducción, síntesis, } \\ \text { análisis) }\end{array} \]

Gráfica 1: Ruta metodológica del proyecto (segunda fase) Fuente: Elaboración propia.

Fases del proyecto (estado actual), en la primera fase del proyecto se realizó una búsqueda de antecedentes con relación a los ejes temáticos fundamentales: las competencias digitales y las competencias interculturales. Esta pesquisa arrojó como resultados la pertinencia de la profundización en las otras dos fases, lo que permitió avanzar hacia una herramienta 
que potencia ambos tipos de competencias para los estudiantes de lenguas extranjeras.

En la implementación de la fase dos, que se desarrolla en simultánea con la fase tres, se indagó a una población de 100 estudiantes de diferentes semestres, pertenecientes al Programa de Lenguas Modernas.

Esta tarea se realizó mediante instrumentos de recolección de datos como encuestas, entrevistas y grupos focales, con la finalidad de construir una cartografía o mapa de ruta que permitiera visualizar cuáles son las competencias que se necesitan trabajar con la herramienta pedagógica que se construiría en la fase tres.

La fase tres del proyecto está determinada por el desarrollo de cada uno de los elementos que intervienen en la construcción del video-juego, en su versión beta. En esta etapa, se hace pertinente la búsqueda de un convenio interinstitucional, ya que la experiencia que puedan aportar otros grupos de investigación ayuda, sin lugar a dudas, a dar mayor sustento a la realización del proyecto y a posibilitar el desarrollo de competencias determinadas para esta primera presentación del video-juego educativo.

\section{Resultados parciales}

En el transcurso de la investigación y hasta el momento de la puesta en marcha de la segunda fase, se han evidenciado significativos avances que han contribuido a identificar las competencias objeto de estudio y a proponer unas estructuras que deben tenerse en cuenta a lo largo de la implementación del proyecto.

En la medida en que existe una alta incidencia comunicativa entre investigadores, se propuso la exploración de las asignaturas de Lengua Materna a través del currículo y, en consecuencia, la generación del esquema sobre competencias comunicativas relacionadas con las necesidades que se presentan en las personas que aprenden una lengua extranjera y paralelamente afianzan el dominio en el español. La gráfica 2 evidencia el proceso de conceptualización de la observación de las nuevas capacidades que un sujeto debe desarrollar en el actual entorno globalizado. En este, se exige la construcción de conocimiento a través de diferentes formas de lenguaje, mediante la concienzación de elementos presentes en la realidad de determinados contextos a los cuales se está avocado en la actualidad.

Competencias comunicativas

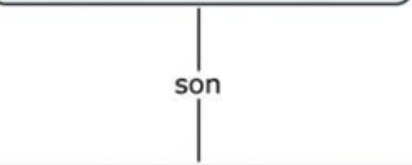

Habilidades de comunicación asertiva

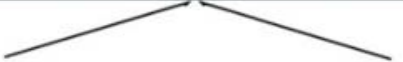

que se logra a través de que tiene en cuenta

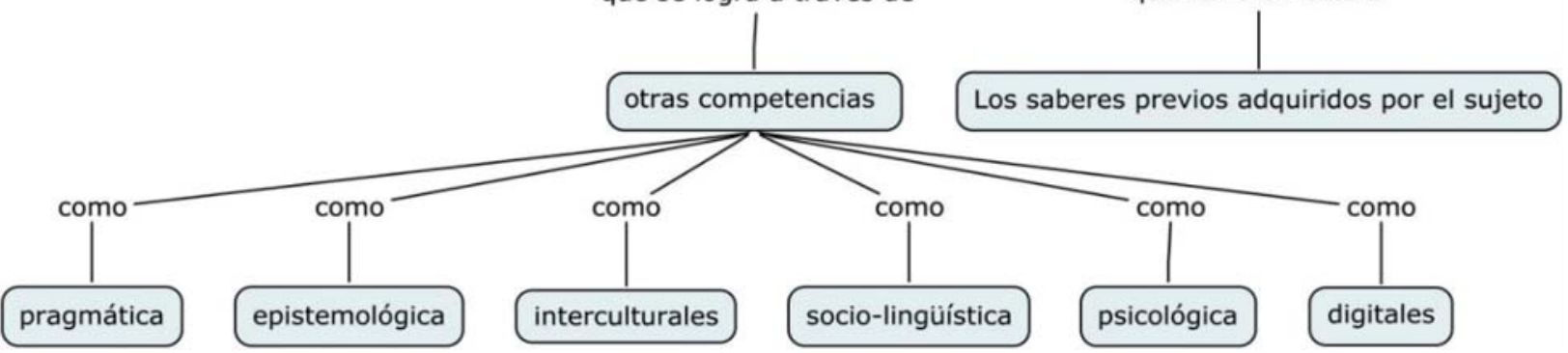


El avance en este sentido demanda el dominio de ciertas habilidades que potencien la relación de conceptos aplicados en situaciones, comunicaciones y contextos lingüísticos específicos, donde los saberes adquiridos puedan hacer parte del desarrollo de una interlocución asertiva.

Por consiguiente, cada una de las competencias propuestas debe proveerse de herramientas con las cuales el individuo pueda desempeñarse de manera adecuada a nivel comunicativo.

También se hace necesario identificar los aspectos tecnológicos que deben tenerse en cuenta en el proceso de formación de profesionales multilingües e integrales, que sean competetitivos en el dominio de herramnientas digitales y puedan comunicarse positivamente a través de ellas.

En ese sentido, la grafica 3 permite observar una cartografía de las competencias digitales que se han considerado para proponer alternativas significativas de aprendizaje.

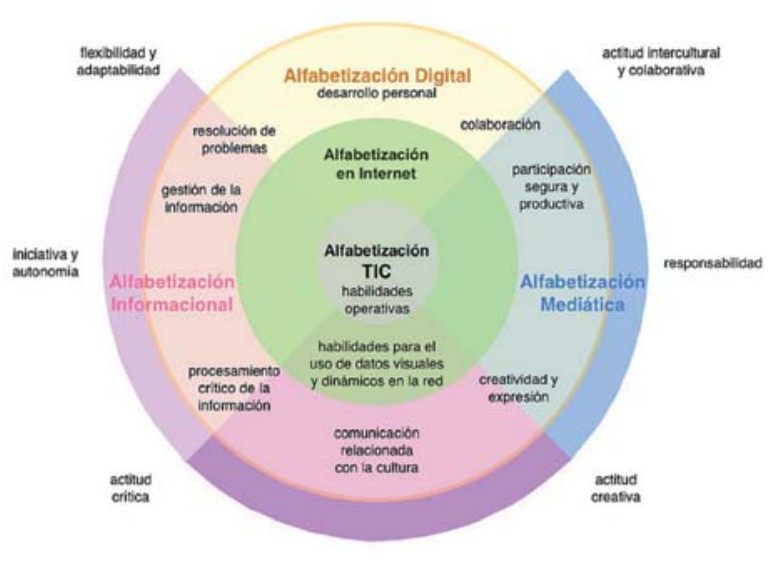

Gráfica 3: Mapping digital competences

Fuente: Ala-Mutka, Kirsti (2011, p. 44).

El dominio de los metalenguajes que se despliegan a partir de lo digital cobra gran relevancia en el aspecto tecnológico, ya que este medio proporciona nuevas herramientas de aprendizaje y fomentan el desarrollo de formas innovadoras de adquirir y ampliar los conocimientos. Por esta razón, cada una de la competencias propuestas por Ala-Mutka (2011) se tornan fundamentales a la hora de relacionarlas con propuestas como la realizada por esta investigación. Aquí se muestra una visión incluyente e integral, tanto de lo tecnológico como de lo comunicacional, y se adjunta lo intercultural como un aspecto que ayuda a cohesionar cada uno de los elementos presentes dentro de la investigación. La gráfica 4 ayuda a percibir las diferentes dimensiones del aprendizaje, según se explica aquí.

\begin{tabular}{|l|l|l|}
\hline \multicolumn{1}{|c|}{ Dimensión } & \multicolumn{1}{|c|}{ Saber } & \multicolumn{1}{|c|}{ Descripción } \\
\hline Instrumental & Acceder a la información & $\begin{array}{l}\text { Adquirir las habilidades instru- } \\
\text { mentales para las búsquedas } \\
\text { de informacion y uso de las } \\
\text { tecnologías }\end{array}$ \\
\hline Cognitiva & $\begin{array}{l}\text { Transformar la información en } \\
\text { conocimiento }\end{array}$ & $\begin{array}{l}\text { Saber plantear problemas. } \\
\text { Analizar e interpretar con } \\
\text { significado la informacòn }\end{array}$ \\
\hline Sociocomunicacional & Expresarsey comunicarse & $\begin{array}{l}\text { Disponer de las habilidades } \\
\text { para crear documentos, di- } \\
\text { fundirlos e interaccionar so- } \\
\text { cialmente }\end{array}$ \\
\hline Axiológica & Usar democrática y éticamente la & $\begin{array}{l}\text { Desarrollo de actitudes, valo- } \\
\text { res y prácticas sociales éticas }\end{array}$ \\
\hline
\end{tabular}

Gráfica 4: Dimensiones del aprendizaje Fuente: Área (2010, p. 46).

Aunado a lo anterior está la propuesta de Área (2010, p. 46), que evidencia la importancia del desarrollo axiológico. Este no se alcanza, en gran medida, dado que la dimensión en la cual se ha centrado el desarrollo de dichas habilidades se limita a lo instrumental y lo sociocomunicacional. En este aspecto, el análisis se presenta con relación a los resultados obtenidos después de la aplicación de los instrumentos de recolección de datos administrados a estudiantes del Programa de Lenguas Modernas de la Escuela Colombiana de Carreras Industriales.

El tercer elemento de estudio se centra en el concepto de la interculturalidad. En la actualidad existen nuevas lecturas de la educación y su contexto, lecturas que de manera transversal analizan la diversidad cultural y los múltiples y complejos procesos políticos, sociales, económicos e inclusive de construcción de imaginarios y representaciones de los individuos; todos ellos, elementos fundamentales en la cons- 
trucción de la sociedad. Y es de estas nuevas lecturas como surgen los estudios interculturales como un medio para promover relaciones positivas entre distintos grupos culturales, de confrontar la discriminación, racismo y exclusión, de formar ciudadanos conscientes de las diferencias y capaces de trabajar conjuntamente en el desarrollo del país y en la construcción de una democracia justa, igualitaria y plural. (Walsh, 2005, p. 8).

Además, de acuerdo con Walsh (1998, p. 119):

La interculturalidad no puede ser reducida a una simple mezcla, fusión o combinación híbrida de elementos, tradiciones, características o prácticas culturalmente distintas. Más bien, la interculturalidad representa procesos (no productos o fines) dinámicos y de doble o múltiple dirección, repletos de creación y de tensión y siempre en construcción; procesos enraizados en las brechas culturales reales y actuales, brechas caracterizadas por asuntos de poder y por las grandes desigualdades sociales, políticas y económicas que no nos permiten relacionarnos equitativamente, y procesos que pretenden desarrollar solidaridades y responsabilidades compartidas.

La interculturalidad cumple un rol crítico, central y prospectivo en todas las instituciones de la sociedad en donde su principal objetivo es reconstruir, de manera progresiva, sociedades, sistemas y procesos educativos, sociales, políticos y jurídicos, así como lograr el reconocimiento de las diferencias y la convivencia democrática.

En ese contexto se entiende por competencia intercultural la habilidad del aprendiente de una segunda lengua o lengua extranjera, para desenvolverse adecuada y satisfactoriamente en las situaciones de comunicación intercultural que se producen con frecuencia en la sociedad actual, caracterizada por la pluriculturalidad. La indagación realizada, pues, ha facilitado la elaboración de una propuesta que integra todos los aspectos referenciados en el documento: lo intecultural, lo digital y lo comunicacional, presentes en este documento como competencias a desarrollar.

Allí se plasman cada uno de los elementos necesarios planteados para la construcción y desarrollo de la fase tres del proyecto de investigación que se expone.

\section{Competencias a desarrollar}

A lo largo de la exposición se ha indicado que la adquisición de diferentes competencias es vital. Por ello se propone que esta primera versión del juego desarrolle competencias comunicativas, interculturales y digitales.

En efecto, el video-juego se presenta como una alternativa educativa, por medio de la cual se conjugan elementos que posibilitan la creación de conocimiento y el perfeccionamiento de competencias para que los estudiantes aprendan conceptos, gestionen nuevos saberes y los intercambien con sus pares, en pro del perfeccionamiento de las habilidades propias en su campo de estudio.

\section{Competencias interculturales}

Estas competencias se construyen con el fin de permitir que el juego contribuya con herramientas que habiliten a las personas para desenvolverse de manera adecuada en los diferentes contestos culturales en los que estén insertos. De acuerdo con los planteamientos propuestos por Altay (2000, p. 51), las competencias a lograr son las siguientes:

1. Capacidad de negociar: incluye aspectos que hacen parte del plano axiológico de los seres en diferentes niveles:

- Mediación conceptual (nivel comunicativo)

- Integración y participación (nivel actitudinal)

- Introspección íntima 
2. Capacidad de gestionar contradicciones simbólicas que se presentan como un reconocimiento del entorno de desenvolvimiento del sujeto:

- lintegración de referentes culturales diferentes

- Gestión de la identidad en la diferencia

- Manejo de contradicciones en valores y proyectos y la descentralización cultural

3. Capacidad de identificar el pluralismo y los comportamientos culturales en contextos sociales; tiene como centro el reconocimiento de las particularidades de otros sujetos con los que se comunica.

Para ello se tienen en cuenta:

- La empatía y la escucha

- La alteridad

- Sensibilidad frente a la diferencia

\section{Competencias digitales}

El vertiginoso crecimiento de las tecnologías de la información ha obligado a que se deban desarrollar habilidades que permitan a los individuos comprender los metalenguajes implícitos en la red global. Ante esta necesidad, aquí se formula una propuesta de competencias relacionadas con el ámbito digital.

Las capacidades digitales que se prevén serán promovidas con la implementación del juego. Se toma como base la taxonomía digital de Bloom, según la propuesta de Andrew Churches (2009, p. 10). Los términos de relevancia a tener en cuenta son:

Evaluar y analizar. El primero incluye los procesos mentales superiores de revisar, formular hipótesis, criticar, experimentar, juzgar, probar, detectar, monitorear, comentar en un blog, revisar, publicar, moderar, colaborar, participar en redes (networking), reelaborar, probar.

Respecto al segundo, se incluyen los procesos mentales de comparar, organizar, deconstruir, atribuir, delinear, encontrar, estructurar, integrar, recombi- nar, enlazar, validar, hacer ingeniería inversa (inverse engineering), "cracking", recopilar información de medios (media clipping).

\section{Competencias comunicativas}

En el desarrollo del análisis de los diferentes elementos pertinentes, se elaboró una propuesta con la cual se puede empezar a abordar el estudio de los aspectos relevantes a la hora de determinar las competencias necesarias de un hablante de segunda lengua. Fruto de esta investigación es la propuesta del modelo que se presenta a continuación y del cual se propone considerar las competencias pragmática, sociolingüística y digital.

La primera —competencia pragmática- hace referencia al desempeño que tiene un sujeto a la hora de comunicarse; capacidad para adecuar el mensaje según las necesidades de enunciación.

La competencia sociolingüística se refiere a los conocimientos de la lengua aplicados en contextos determinados, lo mismo que los usos gramaticales y los aspectos fonéticos y morfosintácticos en dichos contextos. Por último, la competencia digital se hace necesaria ya que al hacer uso de metalenguajes, el dominio de las estructuras de estos permitirá una comunicación asertiva.

Los resultados preliminares permiten la concreción de estrategias metodológicas que faciliten poner en contexto las competencias mencionadas, a través de la gestión de la tecnología y partiendo de las necesidades propias del contexto de estudio.

De este modo, la propuesta busca espacios de reflexión y producción de conocimientos, de forma que la integralidad en la formación de hablantes de una lengua extranjera pueda optimizarse mediante las herramientas tecnológicas que proporcionen las habilidades que necesita y exige la sociedad en red en la que vivimos.

De esta manera, las competencias referenciadas se hacen esenciales a la hora de formular herramientas o metodologías que ayuden a que todos aquellos que 
se acercan a una segunda lengua puedan potenciar su aprendizaje y su posterior desenvolvimiento en el campo laboral. Se parte, entonces, de una comunicación asertiva y de un proceso de interacción que reconoce al otro como elemento fundamental para la generación se conocimiento.

\section{REFERENCIAS BIBLIOGRÁFICAS}

[1] Ala Mutka, K. (2011). Mapping Digital Competence: Towards a Conceptual Understanding. JRC 67075 - Joint Research Centre - Institute for Prospective Technological Studies, http://ftp.jrc.es/EURdoc/ JRC67075_TN.pdf. (26 Octubre 2013)

[ 2 ] Ala Mutka, K.; Punie, Y. \& Redecker, C. (2008). Digital Competence for Lifelong Learning. European Commission, Institute for Prospective Technological Studies, http://ftp.jrc.es/EURdoc/JRC48708.TN.pdf. (26 Octubre 2013)

[3] Altay, M. (2000). Compétences interculturelles et stratégies identitaires, Agora débats jeunesses, 22. INJEP-L'Harmattan, Marlyle-Roi, pp. 49-6o.
[ 4 ] Área, M. (2010). Aprender a buscar y analizar información, aprender a reelaborar y difundir información, aprender a expresarse y comunicarse,http:// www.aab.es/app/download/6054131/Bibliotecas-Bolet\%C3\%ADng8-99.pdfdDoc.csic. es:808os/app/down-load/6054131/BibliotecasBolet\%C3\%ADng8-99.pdf. (17 Noviembre 2013)

[ 5 ] Churches, A. (s.f.). Taxonomía de Bloom para la era digital, http://www.eduteka.org/TaxonomiaBloomDigital.php. (26 de Octubre 2013)

[6] Hernández, S. (2006). Metodología de la investigación. México DF: Pearson.

[ 7 ] Walsh, C. (2005). La interculturalidad en la educación. Lima, Perú: Ministerio de Educación.

[8] Walsh, C. (1998). La interculturalidad y la educación básica ecuatoriana: Propuestas para la reforma educativa, Procesos. Revista Ecuatoriana de Historia, 12, pp. 119-128. 
